\title{
Nuevos relatos más accesibles. "A peu de Museu", un aplicativo web del Museo de Cervera
}

\author{
New more accessible stories. "A peu de Museu", a web application of \\ the Museum of Cervera
}

\section{Carme Bergés Saura}

Museu de Cervera carmeberges@gmail.com

\begin{abstract}
Resumen
'A Peu de Museu' es una invitación a recorrer las calles de Cervera desde una nueva perspectiva. La tecnología de nuestro móvil nos permitirá entrar en 3 historias protagonizadas por 3 mujeres que representan 3 momentos clave de la historia de la ciudad: el pasado medieval, judio y barroco.

Para el Museo, el proyecto es una oportunidad única para "colonizar" la ciudad y explicar "in situ" su historia; en definitiva, es una oportunidad para romper los muros de la institución y ofrecer significados relevantes que conectan intelectualmente y emocionalmente. Es importante también la aplicación de la perspectiva de género: las protagonistas son 3 mujeres que no solo representan 3 momentos históricos, sino también 3 condiciones sociales. Margarita, la mujer medieval, fue la esclava del señor de Menarguens. Regina, la protagonista judí, formaba parte de una familia poderosa que deberá lidiar con una férrea tradición. Ignacia Brach, regentó una taberna en la rígida Cervera universitaria del siglo XVIII.
\end{abstract}

Las 3 rutas son accesibles en 4 idiomas e incorporan códigos QR que dan acceso a diferentes contenidos. Esta nueva herramienta ofrece un total de 26 puntos de interpretación con la grabación de 65 minutos de locución, más de 200 recursos audiovisuales que incluyen fotografies antiguas y actuales con gifs que permiten descubrir, con un solo movimiento de dedo, los cambios históricos. Contiene también videos, imágenes $360^{\circ}$, fotografias tomadas con drone y la visualización de más de 50 obras de la colección del Museo (con la previsión de actualizaciones futuras).

Todos estos recursos digitales se organizan en apartados, que pueden ser consultados libremente según la motivación o el tiempo disponible. La locución narra en primera persona la vida y sentimientos de la protagonista y sitúan a los visitantes en un espacio vivido, el "Cuaderno del viajero" ofrece 
explicaciones más académicas y objetivas de los elementos patrimoniales, la 'Historia Gráfica', ayudará a reconstruir el pasado a partir de fotografías y/o documentos, y la 'Galería del Museo' muestra los objetos custodiados per el Museo provocando el interés por visitar la institución.

Este proyecto es una iniciativa del Museo de Cervera, con la colaboración de la Agència Catalana del Patrimoni Cultural de la Generalitat de Catalunya. Con el confinamiento ha sido una de las herramientas más utilizadas ya que todos los contenidos de la aplicación web se pusieron en abierto para su utilización desde casa.

Palabras clave: Tecnología aplicada al patrimonio, perspectiva de género, rutas históricas $Q R$, interpretación del patrimonio, relatos inclusivos.

\footnotetext{
Abstract

'A Peu de Museu' is an invitation to walk the streets of Cervera from a new perspective. The technology of our mobile will allow us to enter 3 stories starring 3 women who represent 3 key moments in the history of the city: the medieval, Jewish and baroque past.

For the Museum, the project is a unique opportunity to "colonize" the city and explain its history "in situ"; in short, it is an opportunity to break down the walls of the institution and offer relevant meanings that connect intellectually and emotionally. The application of the gender perspective is also important: the protagonists are 3 women who not only represent 3 historical moments, but also 3 social conditions. Margarita, the medieval woman, was the slave of the Lord of Menarguens. Regina, the Jewish protagonist, was part of a powerful family that must deal with an iron tradition. Ignacia Brach, ran a tavern in the rigid university Cervera of the eighteenth century.
}

The 3 routes are accessible in 4 languages and incorporate $Q R$ codes that give access to different content. This new tool offers a total of 26 points of interpretation with the recording of 65 minutes of speech, more than 200 audiovisual resources that include old and current photographs with gifs that allow you to discover, with a single movement of the finger, historical changes. It also contains videos, $360^{\circ}$ images, photographs taken with drone and the visualization of more than 50 works from the Museum's collection (with the anticipation of future updates).

All these digital resources are organized into sections, which can be freely consulted depending on the motivation or the time available. The locution narrates in the first person the life and feelings of the protagonist and places the visitors in a lived space, the "Traveler's Notebook" offers more academic and objective explanations of the heritage elements, the 'Graphic History', will 
help to reconstruct the passed from photographs and / or documents, and the 'Gallery of the Museum' shows the objects guarded by the Museum, provoking interest in visiting the institution.

This project is an initiative of the Cervera Museum, with the collaboration of the Catalan Agency for Cultural Heritage of the Generalitat de Catalunya. With the confinement it has been one of the most used tools since all the contents of the web application were made open for use from home.

Keywords: Technology applied to heritage, gender perspective, historical $Q R$ routes, heritage interpretation, inclusive stories. 


\section{Introducción}

El Museo de Cervera está inmerso en un doble proceso de reflexión y de cambio. Por un lado, lleva a cabo una reflexión que analiza y cuestiona cuál es —o debería ser- la posición del museo en relación con la ciudad donde se ubica, y su papel e implicación en las transformaciones de sitios y relaciones entre las comunidades que los habitan. Se trata de una mirada introspectiva y crítica hacia la propia institución, que necesita romper los muros del edificio para explicar la ciudad más allá de las colecciones que conserva y que, en su mayor parte, se encuentran almacenadas a causa de una ampliación arquitectónica y museográfica que nunca llega. Una necesidad, por lo tanto, de expandirse hacia la "vida", salir a la calle y desarrollar nuevos discursos que, inevitablemente, deben trascender los objetos, aunque partan de ellos, para explicar la ciudad, plural y diversa, y especialmente para establecer nuevas perspectivas que nos ayuden a entender nuestro presente (Plan Estratégico del Museo de Cervera, 2021-2025).

Por otro lado, desarrolla un cambio global que se enmarca en la tendencia actual hacia el nuevo concepto de museo social, un museo que se aleja de la visión tradicional, que prioriza el contenido y los objetos y que lidera procesos unidireccionales, para reivindicar la presencia de los públicos, su participación y su relación real con la institución. Es decir, unos museos preocupados por establecer comunicaciones multidireccionales y por crear experiencias de aprendizaje competencial. Huelga decir que estos objetivos pueden valerse de la combinación de objetos tangibles o digitales, del trabajo hecho a partir de la cocreación de contenidos y de la introducción de nuevas narrativas sociales, inclusivas y accesibles (ILMS, 2009).

Esta tendencia, que lleva años fraguándose, hasta remover y forzar la propia definición de museo (https://icom.museum/es/news/el-icom-anuncia-la-definicion-alternativa-del-museoque-se-sometera-a-votacion/), ha sido liderada, seguramente de forma invisible, por buena parte de los pequeños museos (¿de territorio?, ¿de sociedad?) dispersos por nuestro territorio, con estructuras y financiación insuficientes, pero que han reivindicado históricamente su función social y su implicación en el desarrollo local. Han abanderado la idea de servicio público y han concentrado sus esfuerzos en crear vinculación, aun con una infraestructura obsoleta y un equipo profesional reducido al mínimo.

En estos momentos, a consecuencia de la situación excepcional provocada por la pandemia, esta tendencia se ha visto acentuada y acelerada, pero era un cambio anunciado a gritos. La digitalización como herramienta de interpretación, la expansión del museo más allá de sus muros, la accesibilidad de contenidos, la democratización de la cultura, la necesidad de experimentar, la búsqueda de nuevas narrativas que nos unan, y la oportunidad de participar en la construcción de relatos son algunas de las demandas a las que los museos debemos dar respuesta. La tecnología puede ser un medio eficaz, aunque no debe monopolizar nuestras acciones. Ahora más que nunca, la tecnología plantea soluciones híbridas, mixtas y diversas 
para adecuarse a públicos (físicos y digitales) también diversos, para construir contenidos complejos y para afrontar nuevos retos de accesibilidad, movilidad e inclusión.

La dimensión digital, por lo tanto, no responde a un único objetivo de difusión, de mejora del conocimiento, de accesibilidad, de comunicación o de educación, sino que lo impregna absolutamente todo: desde los procesos hasta los resultados.

Así pues, esta doble reflexión nos ha llevado a elaborar nuevos recursos que deben responder a una clara voluntad de innovación, de expansión más allá de los muros y de reivindicación de nuevos protagonismos (sobre quién escribe la historia — perspectiva de género-y sobre cómo se escribe y se investiga —utilización de otras fuentes o la relevancia de la microhistoria-). La tecnología no ha sido la respuesta a todas estas necesidades, pero sí se ha convertido en su herramienta.

En este contexto de planificación, y después de varios años de investigación, el museo estrenaba una nueva aplicación web que tenía que cumplir con muchas de las premisas hasta ahora presentadas. En 2019, coincidiendo con la celebración de Cervera Capital de la Cultura Catalana, el Museo de Cervera consiguió trabajar juntamente con la Agència Catalana de Patrimoni de la Generalitat de Catalunya en un proyecto que reunía objetivos comunes: acercar el patrimonio a la ciudadanía, hacerlo utilizando la tecnología como vehículo de interpretación y apoyar las iniciativas surgidas del propio territorio donde debía desarrollarse la acción.

Así nacía “A Peu de Museu”, un aplicativo para móvil con un título intencionado, que juega con la expresión "a pie de calle", o aquello que encuentras de forma fácil y directa, con un guiño a su misión principal, la accesibilidad.

\section{Objetivos}

Aunque en la introducción de este texto, en parte, ya se han esbozado algunos de los objetivos fundamentales de este nuevo recurso, vamos a intentar sintetizar los principios que lo guiaron.

En relación con la herramienta digital, se pretendía:

-HD1. Crear una herramienta que ayude a mejorar el conocimiento. En nuestro caso, de Cervera, mostrando la construcción y evolución de la ciudad, en sus dimensiones política, social y urbanística y desde el punto de vista de las personas y su cotidianidad.

-HD2. Facilitar el acceso a la información. Era necesario experimentar con nuevos formatos que permitieran "traducir" todos los contenidos que la investigación especializada y rigurosa nos aportaba en un lenguaje demasiado académico y convencional.

En este sentido, cabe destacar un aspecto para nosotras fundamental: la investigación y los contenidos son el fundamento, nuestra materia prima para crear recurso interpretativo, sea del tipo que sea, pero cada recurso precisa de una $u$ otra información, perspectiva y 
tratamiento. Así pues, es necesario apoyarse en la investigación para elaborar contenidos específicos y pensados desde el inicio para el canal que los transmitirá (Rodà, 2012).

-HD3. Facilitar el acceso a los contenidos del museo desde cualquier parte del mundo y en cualquier momento. Aunque en el caso del Museo de Cervera, podríamos pensar que nuestro público es mayoritariamente local, hay que pensar en grande. Hay que romper barreras físicas y lanzar ofertas culturales con un sentido glocal. La situación provocada por la pandemia ha demostrado, de forma contundente, que necesitamos más canales de difusión que nos permitan el acceso a la información desde cualquier parte, pero también que combinen lenguajes, estilos y tratamientos que favorezcan la interpretación y el (re)conocimiento.

-HD4. Animar a conocer el museo. El Museo de Cervera se encuentra en una situación compleja, provocada por el cierre provisional de su colección histórica. Aunque es cierto que el museo se caracteriza por su dinamismo en la programación de exposiciones temporales y actividades que respondan a las demandas de su comunidad, las colecciones históricas se encuentran parcialmente cerradas. Este hecho debilita la posición del museo, que ve mermada su oferta y, consecuentemente, sus nodos de atracción. Por lo tanto, detrás del proyecto "A Peu de Museu" hay también un objetivo dirigido a aumentar los públicos del museo, tanto físicos como digitales.

-HD5. Romper los muros del museo y sacar sus colecciones a la calle. La situación descrita en el punto anterior enlaza con el presente objetivo, pero no lo explica en su totalidad. De hecho, la necesidad de romper los muros físicos del museo y salir a la calle (de ahí también, tal como ya hemos apuntado en la introducción, el juego de palabras con el nombre de la aplicación web) es el resultado de una reflexión profunda sobre la función social que debe jugar el museo. Uno de los retos clave del Museo de Cervera es la dinamización del centro histórico, y en particular de la calle Mayor, donde se ubica la sede principal, actualmente en situación de decadencia. El museo debe actuar como centro de servicios y de interpretación, para mostrar la ciudad al turismo cultural, y también como espacio de conocimiento, reflexión y participación sobre el futuro de la ciudad. Proyectos que aporten nuevas visiones y conocimiento sobre la ciudad no solo ayudan al desarrollo del lugar, sino que también posicionan el museo en la comunidad y entre los visitantes (Plan Estratégico del Museo de Cervera, 2021-2025).

-HD6. Incitar a explorar contenidos más profundos (Stack, 2013) y presentarlos en diferentes niveles. El aplicativo web "A Peu de Museu" se basa en una estructura progresiva de contenidos (que veremos en detalle en el apartado siguiente) pensada para que el usuario pueda decidir en todo momento su grado de implicación, el tiempo de dedicación, la ampliación de información requerida o, también, la selección de información según el perfil, las edades o las motivaciones.

-HD7. Crear un equipo multidisciplinar basado en un trabajo horizontal, colaborativo y, a ser posible, vinculado estrechamente con el territorio. Para la elaboración del proyecto se contó con un equipo de diez profesionales para las siguientes tareas: desde la 
elaboración de contenidos por un equipo de tres historiadores/as y un documentalista con funciones de coordinación y de creación de recursos, al diseño de la interfaz, la marca y la señalización interpretativa, la programación informática y su presentación en el sitio web del museo, o a la ilustración y las voces utilizadas para dar vida a las protagonistas de cada historia.

En relación con los contenidos, los museos partimos de una situación de privilegio, ya que nuestras colecciones no son solo objetos, sino que son contenedores de memoria, en toda su complejidad y con un alto potencial para conectar las personas con los sitios. "Los museos conservan objetos, pero también conocimiento, historias, creación, imágenes... y cada una de estas pequeñas piezas puede tener relación con un lugar en el mapa” (Sierra, 2012).

Siendo conscientes de ello, el proyecto "A Peu de Museu" va más allá de unos contenidos concretos y amplía sus objetivos a:

\section{-C1. Construir nuevas narrativas más sociales e inclusivas:}

oAplicación de la perspectiva de género. Nuestras protagonistas son tres mujeres con nombres y apellidos reales, sobre las cuales se ha conseguido trazar un retrato bien documentado que enlaza hechos históricos con la recreación de la propia "vida", la cotidianidad y los sentimientos de unos personajes que bien podríamos ser nosotras mismas. oUtilización de fuentes que nos permiten dibujar la microhistoria, la cotidianidad del momento histórico para crear emoción. El "usuario" puede conectar e implicarse con las historias de vida de las mujeres que narran su periplo vital. Esta conexión facilita el conocimiento, crea vínculos y trabaja sentimientos fundamentales como la empatía, al mismo tiempo que ayuda a la reflexión crítica sobre el lugar, el tiempo y las personas.

\section{-C2. Abordar temas desde lo local para construir relatos globales y, al mismo tiempo, tratar temas históricamente olvidados o silenciados:}

oLa transformación de las ciudades y su (re)conocimiento.

oConflictos sociales: la esclavitud en la Edad Media catalana, la aljama judía y el peso de la tradición, la época del Barroco y la huella de mujeres con empuje y relevancia. 


\section{Desarrollo}

"A peu de Museu" es una aplicación web responsiva con visualización móvil a través de códigos QR que se sitúan en diferentes puntos de la ciudad y que construyen tres rutas temáticas protagonizadas, como ya se ha dicho, por tres mujeres que ayudan a entender el momento y el sitio histórico.

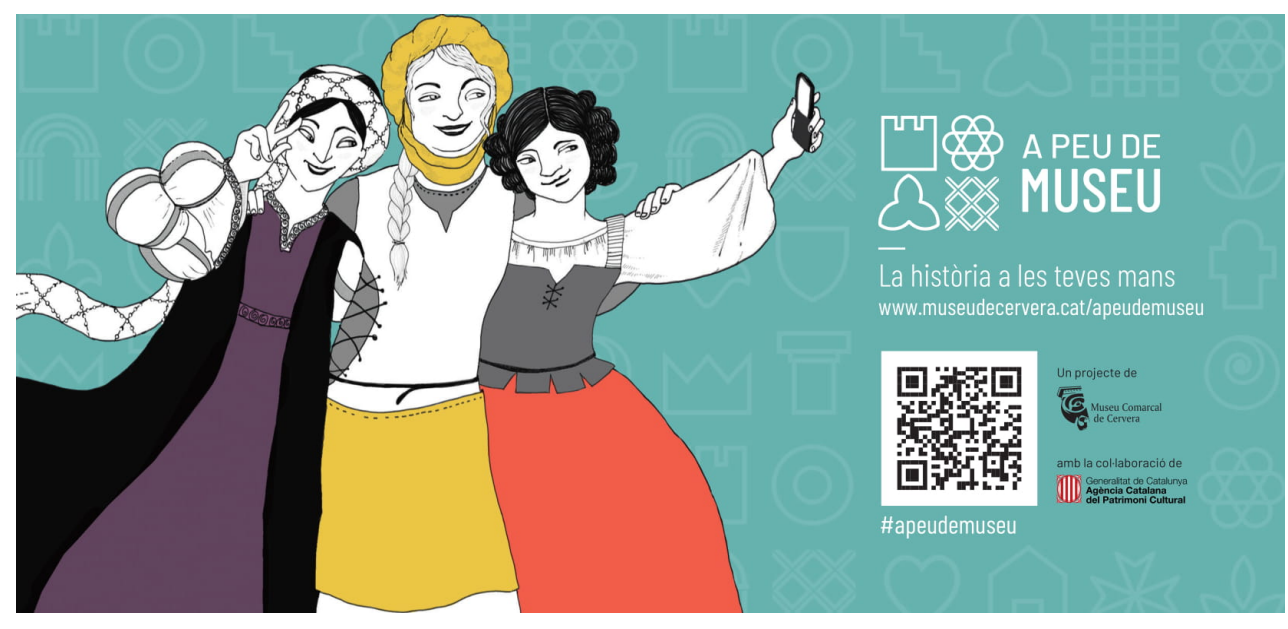

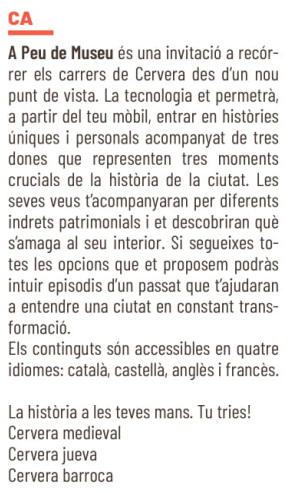

A Peu de Museu és una invitació a recórrer els carrers de Cervera des d'un no úniques i personals acompanyat de tres resenten tres moments seves veus t'acompanyaran per diferents indrets patrimonials $i$ et descobriran què es les opcions que et proposem podràs intuir episodis d'un passat que t'ajudar a entendre una ciutat en constant trans-

idiomes: català, castellà, anglès i francès.

La història a les teves mans. Tu tries.

Cervera medieva

Cervera barroca
EN

A Peu de Museu (The Museum in the Street) is an invitation to explore the streets of Cervera from a new perspective. With technology, in the shape of a mobile phone you can participate in the stories of three women living at three crucial moments in the city's history. Their voices will accompany you on your journey as you visit the town's historic sites and buildings, and they will reveal what is hidden inside. If you follow all the routes, you will witness some events from the past, as if at first hand, and form a view of Cervera as a town in constant transformation. ges: Catalan, Spanish, English and French. History in hour hands! Choose from. Medieval Cervera Jewish Cervera Baroque Cervera

\section{ES}

A Peu de Museu (El museo en la calle) es una invitación a recorrer las calles $d$ Cervera desde un nuevo punto de vista. Gracias a la tecnologia, a través del móvi podréis sumergiros en historias únicas y personales de la mano de tres mujeres que representan tres momentos cruciales e la historia de la ciudad. Sus voces os compan̄arán por diferentes lugares paimoniales y os descubrirán qué se esconde en su interior. Si sequís todas las opciones que se proponen, podréis intuir episodios de un pasado que nos ayudan entender una ciudad en constante tran

Los contenidos están disponibles en catalán, castellano, inglés y francés.

La historia en tus manos. iTú eliges!

Cervera medieval

Cervera judia

Cervera barroca
FR

A Peu de Museu (Le musée dans les rues) est un invitation à parcourir les rues de Cervera avec une nouvelle perspective. À partir de notre téléphone portable, la technologie nous permettra de vivre des histoires uniques et personnelles de la main de 3 femmes reprentont 3 ons

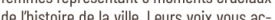
de thistore de la vile. Leurs voix vous acmonix et vous fent cache Sivoss silez que nous vous propo toutses les options que nous con nitro cetains des episades da passe qui nous aident a comprendre une ville en Les contenus sont disponibles en 4 langues: catalan, espagnol, anglais et français.

L'histoire dans vos mains. A vous de choisir! Cervera médiévale

Cervera juive Cervera baroque

Fig. 1 Imagen del díptico diseñado para la difusión del proyecto 


\subsection{Estructura y organización de los contenidos}

El eje vertebrador del proyecto es, sin lugar a duda, el personaje protagonista de cada ruta y su historia de vida. Son ellas quienes acompañan a los visitantes (físicos y digitales), los guían por lugares concretos y los introducen en su historia particular. Para ello, el recurso principal es la voz en primera persona de estas mujeres, a las que se dotó de anécdotas, parentescos, hechos y situaciones concretos para ganar realismo. La investigación se centró en la búsqueda de tres mujeres reales sobre las cuales existiera información relevante y valiosa; las lagunas que pudieran existir en su trayectoria vital fueron rellenadas con hechos documentados que nos permitieron trazar un retrato verosímil. Se completó su biografía con un retrato ilustrado, que ayudaba a hacerla más real y próxima. Estas ilustraciones sirvieron también para presentar las rutas en la señalización urbana y para toda la campaña de difusión y publicidad del proyecto.

\section{CERVERA MEDIEVAL}

Mi nombre es Margarida, esclava de Menarguens. Tengo dos hijos. El primero de un padre desconocido, al menos así se registró para que su nombre no se hiciera público, pero ciertamente sé quién era. Lo bauticé con el nombre de Pere el 21 de abril de 1434 en la iglesia de Santa María. Mi sequndo hijo nació tres años después, a mediados del verano, y se bautizó el 12 de julio. En esa ocasión el cura accedió a registrar el nombre del padre: el maestro Gauter, notario público de profesión y buen amigo de mi maestro, iy ahora también del niño!

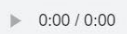

VERRUTA

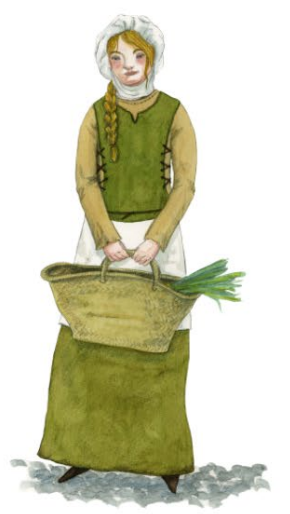

CASTELL DE CERVERA

PLACA VELLAI CONVENT DE SANT DOMĖNEC

ESGLÉSIA DE SANTA MARIA

PLAÇA DEL BLAT O MERCADAL

CARRERÓ DE LES BRUIXES

ESGLÉSIA DE SANT BERNAT I CASA DE SANTES CREUS

CALL JUSSA

CASES MEDIEVALS

PLACA SANTA ANNA 0 PLACA SANTA AN
DE LES BESTIES

ESGLÉSIA DE SANT ANTONI 
Nuevos relatos más accesibles. “A peu de Museu”, un aplicativo web del Museo de Cervera

Mi nombre es Regina y me casé con Issach de Querci, hijo de Issach de Querci mayor, hermano de Jacob. Todo ha ido mal desde que murió mi marido. No teníamos un heredero varón para continuar con el apellido y eso significa, según nuestras leyes, que estoy obligado a casarme con mi cuñado Jacob. Ha huido a la baronia de Anglesola porque no quiere... y en cuanto a mi, inadie me ha preguntado qué pienso! Los Ouercís han tenido que acudir a la cancillería del rey Juana II para hacer cumplir el matrimonio o dejarme libre por medio de halizah. Aunque ninguna de las opciones es satisfactoria, han decidido concederme la libertad.

$0: 00 / 0: 59$

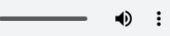

VER RUTA

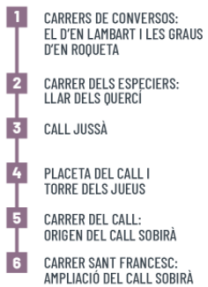

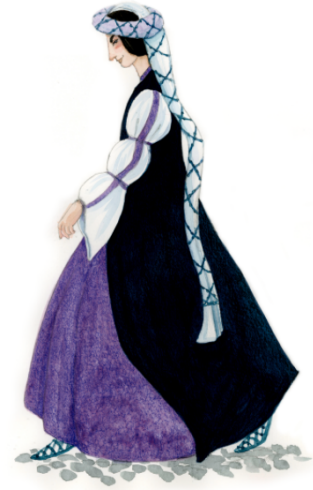

\section{CERVERA BARROCA}

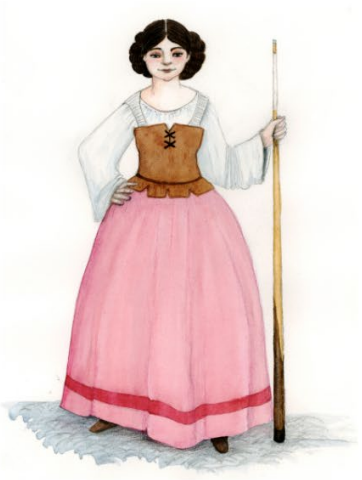

Soy Ignasia Brach , pero todos me conocen como Nasa

Tabaco. Dirijo una posada en la calle Major de Cervera popular entre los estudiantes universitarios y muchos otros jóvenes de la zona. En mi familia hay autoridades de renombre vinculadas a la universidad y saben

exactamente lo que representa mi hostería: un lugar de libertad dentro del rigido régimen universitario de Cervera. A pesar de las frecuentes disputas entre este público joven, me gusta pensar que se sienten como en casa en

mi posada porque yo brindo servicios y juegos que no todos los demás pueden. ithe traído la primera mesa de billar de la ciudad desde Barcelona!

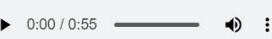

VER RUTA

\begin{tabular}{l|l}
1 & ESGLÉSIA DE SANTA MARIA \\
PAERIA DE CERVERA
\end{tabular}

Fig. 2 Capturas de imagen del web donde se presenta las tres protagonistas y los puntos de interés de cada ruta temática 
A partir de la locución de las historias en primera persona, la aplicación aporta diferentes niveles de información que permiten un acceso a contenidos más profundos sobre el sitio visitado o los hechos narrados. Se utilizaron recursos varios, que incluyen desde fotografías históricas, que permiten ver los cambios en el urbanismo de la ciudad y la ocupación del espacio al largo de la historia, a descripciones de carácter más académico. Uno de los objetivos importantes del proyecto era incentivar la visita al museo, (re)conocerlo y aumentar así sus públicos y, para ello, se habilitó una galería de imágenes que permite la visión de las colecciones del museo que proceden del lugar que se está visitando en cada momento.
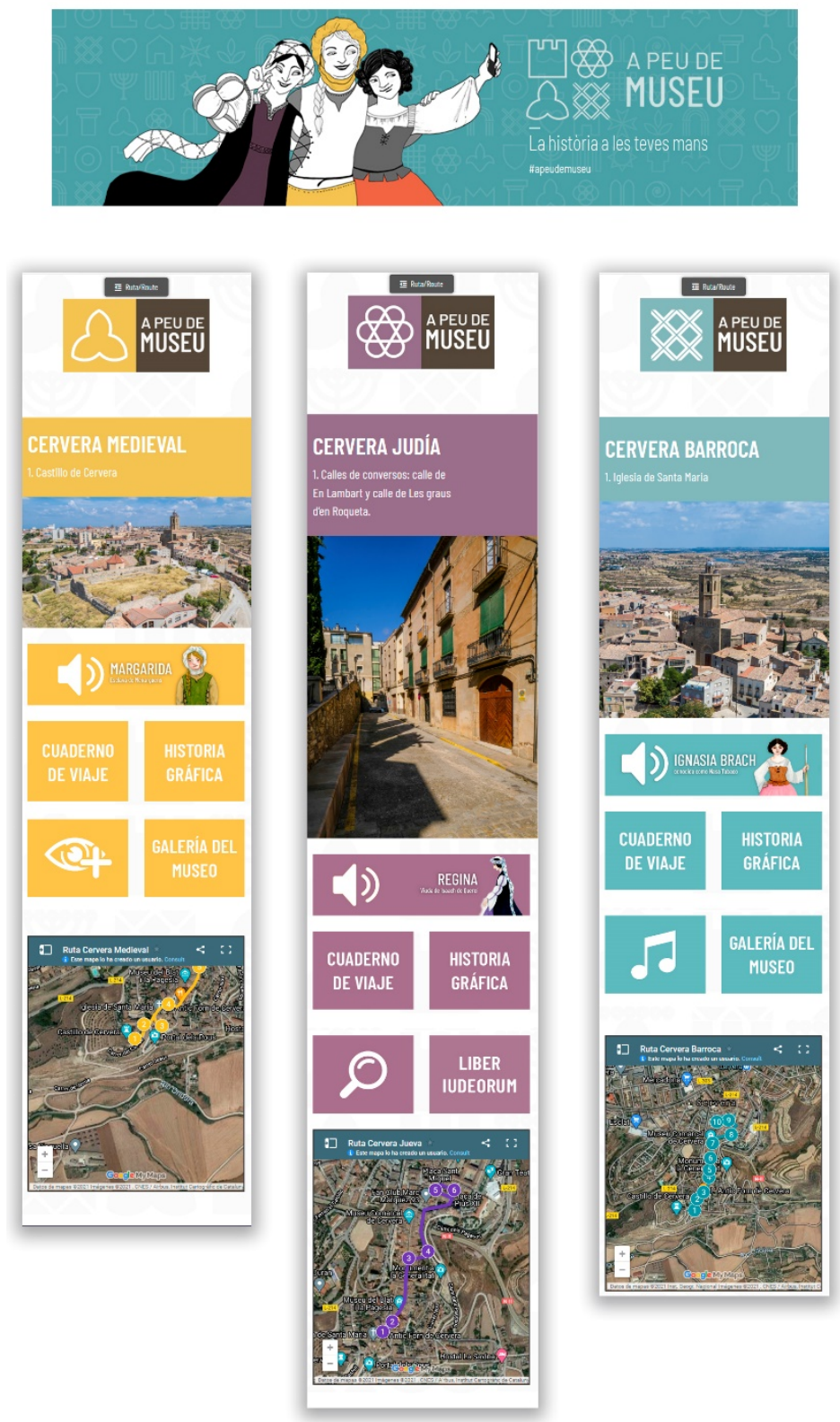

Fig. 3 Captura de imagen del menú con las opciones de contenidos vinculados a las rutas 
En definitiva, se trata de un menú que se convierte en múltiples posibilidades de acceso a informaciones varias y complementarias, pero que pueden ser usadas independientemente, sin perder el hilo del relato principal.

A continuación, presentamos un cuadro resumen de las opciones de menú, el tipo de recurso asociado, el carácter o enfoque que utiliza y los objetivos que persigue:

Tabla 1. Menú

\begin{tabular}{|c|c|c|c|}
\hline MENÚ & RECURSO & ENFOQUE & OBJETIVOS \\
\hline LOCUCIÓN & $\begin{array}{l}\text { AUDIO + BIOGRAFÍA } \\
\text { ESCRITA + RETRATO } \\
\text { ILUSTRADO }\end{array}$ & $\begin{array}{l}\text { Narración en primera } \\
\text { persona + ilustración } \\
\text { subjetiva }\end{array}$ & $\begin{array}{l}\text { HD1, HD2, } \\
\text { HD3, HD6, } \\
\text { HD7 } \\
\text { C1, C2 }\end{array}$ \\
\hline $\begin{array}{l}\text { CUADERNO } \\
\text { DEL VIAJERO }\end{array}$ & $\begin{array}{l}\text { TEXTO ESCRITO } \\
\text { DESCRIPTIVO }\end{array}$ & $\begin{array}{l}\text { Información de } \\
\text { carácter académico }\end{array}$ & $\begin{array}{l}\text { HD1, HD2, } \\
\text { HD3 }\end{array}$ \\
\hline $\begin{array}{l}\text { HISTORIA } \\
\text { GRÁFICA }\end{array}$ & $\begin{array}{l}\text { RECURSOS } \\
\text { GRÁFICOS + } \\
\text { MULTIMEDIA }\end{array}$ & $\begin{array}{l}\text { Documentación + } \\
\text { restitución histórica }\end{array}$ & $\begin{array}{l}\text { HD1, HD2, } \\
\text { HD3, HD5, } \\
\text { HD6, HD7 } \\
\text { C1 }\end{array}$ \\
\hline $\begin{array}{l}\text { GALERÍA DEL } \\
\text { MUSEO }\end{array}$ & $\begin{array}{l}\text { RECURSOS } \\
\text { GRÁFICOS + } \\
\text { INFORMACIÓN } \\
\text { BÁSICA }\end{array}$ & $\begin{array}{l}\text { Las colecciones del } \\
\text { museo en imágenes }\end{array}$ & $\begin{array}{l}\text { HD1, HD2, } \\
\text { HD3, HD4, } \\
\text { C1, C2 }\end{array}$ \\
\hline OTROS & $\begin{array}{l}\text { VISITAS VIRTUALES } \\
+ \\
\text { RUTAS } \\
\text { ALTERNATIVAS }\end{array}$ & $\begin{array}{l}\text { Documentación + } \\
\text { restitución histórica }\end{array}$ & $\begin{array}{l}\text { HD1, HD2, } \\
\text { HD3, HD5, } \\
\text { HD6, HD7 }\end{array}$ \\
\hline
\end{tabular}

Fuente: elaboración propia 


\subsection{La creación de marca}

Si bien el contenido es la parte esencial, el esqueleto que sostiene cualquier proyecto cultural, el diseño y los soportes de difusión tienen gran importancia, ya que aportan valor y atractivo, lo que garantiza su éxito.

Por eso, desde el planteamiento inicial del aplicativo web, se incorporó en el equipo una diseñadora, que construyó, en diálogo con el resto del equipo — documentalistas, historiadores/as e informáticos_-, la marca que identifica todo el proyecto:

\section{- Logotipo y sus aplicaciones en web, papelería y señalización}
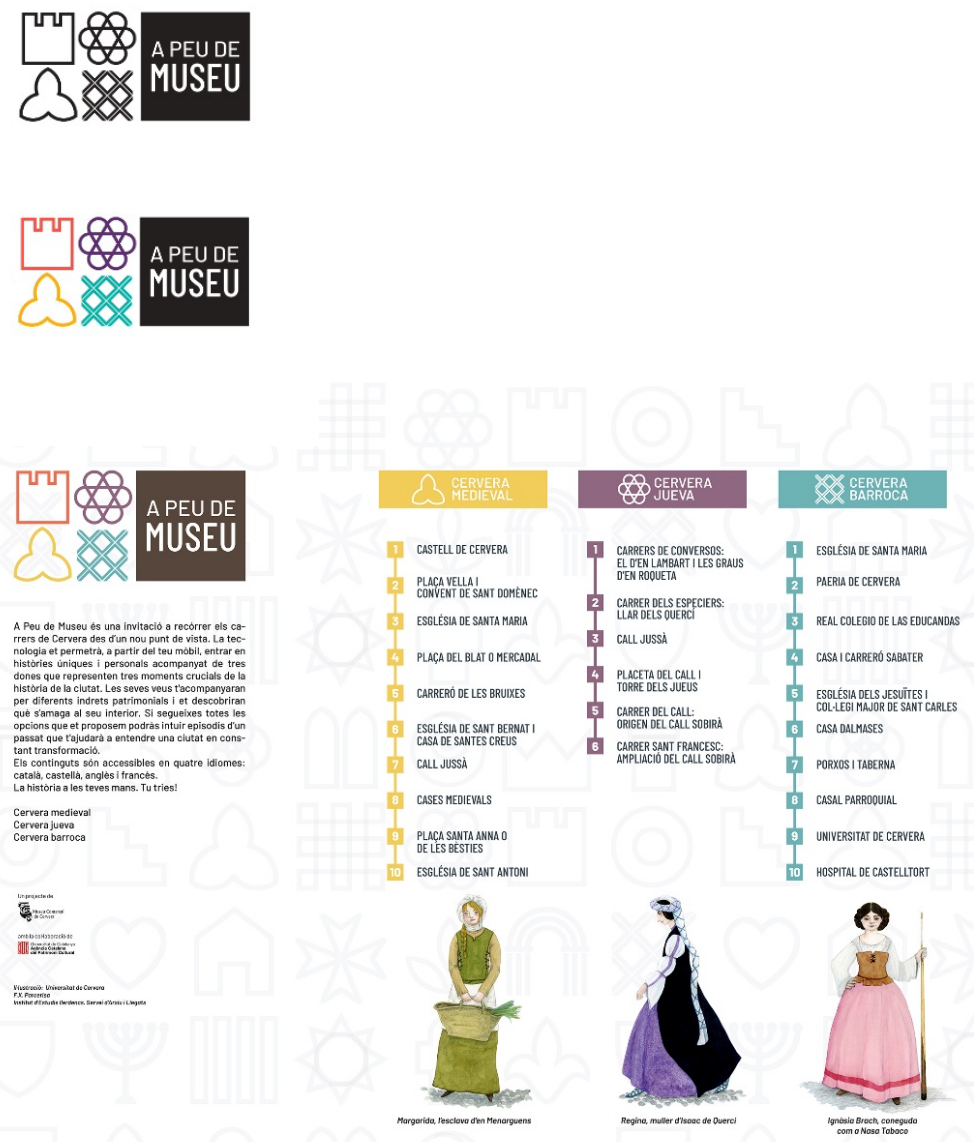

Fig. 4 Imagen del diseño del logotipo y sus diferentes aplicaciones 


\section{- Señalización interpretativa}

Se crearon dos tipos de señalización:

- Placas con el código QR que enlaza con la web de contenidos. Se hallan delante del punto de interés y se identifican según el color y la marca distintiva de cada ruta. De este modo, el seguimiento de la ruta es más visual e intuitivo $\mathrm{y}$, al mismo tiempo, permite visibilizar rápidamente aquellos puntos comunes a más de una ruta o, lo que es lo mismo, ver como un mismo sitio puede ser "leído" desde momentos históricos distintos.

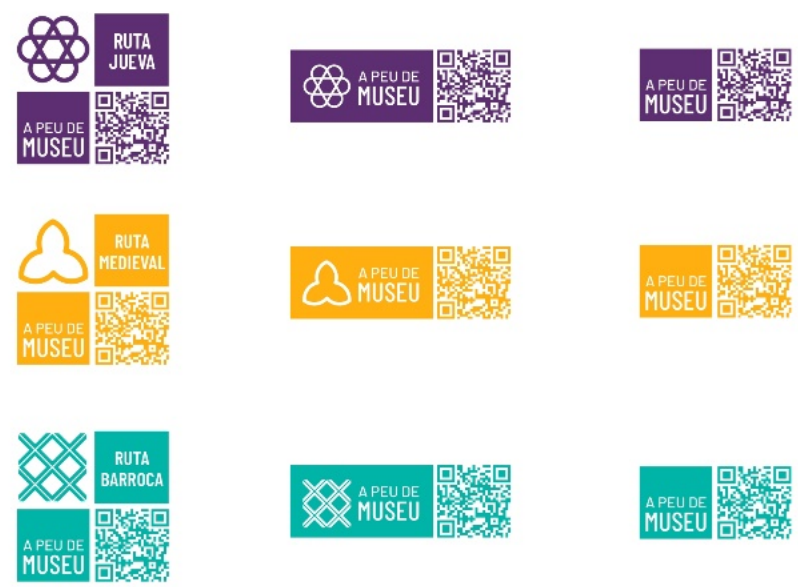

Fig. 5 Imagen del diseño de la señalización QR urbana

- Señalización interpretativa de gran formato situada en puntos estratégicos del tejido urbano. Estas estructuras cumplir dos objetivos básicos: aportan información práctica sobre las rutas $\mathrm{y}$, al mismo tiempo, reproducen una fotografía histórica sobre el lugar donde se ubican para convertirlo en un "lugar vivido". 


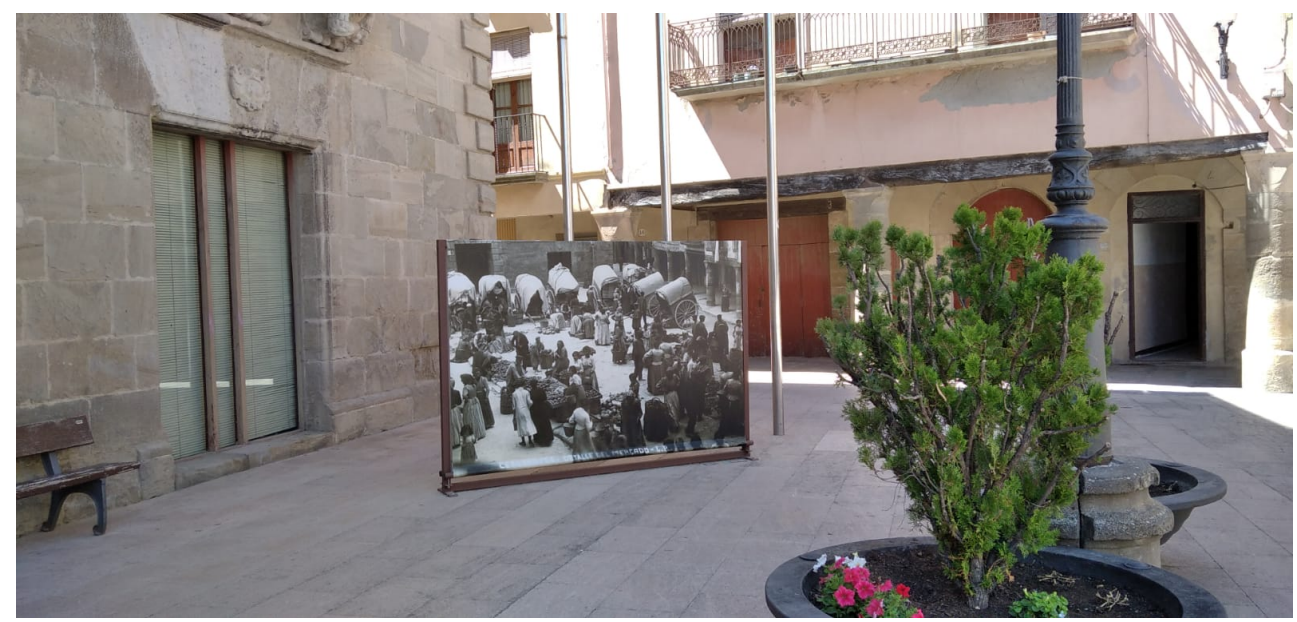

Fig. 6 Señalización interpretativa con la imagen histórica a gran formato de la plaza Mayor durante la celebración del mercado

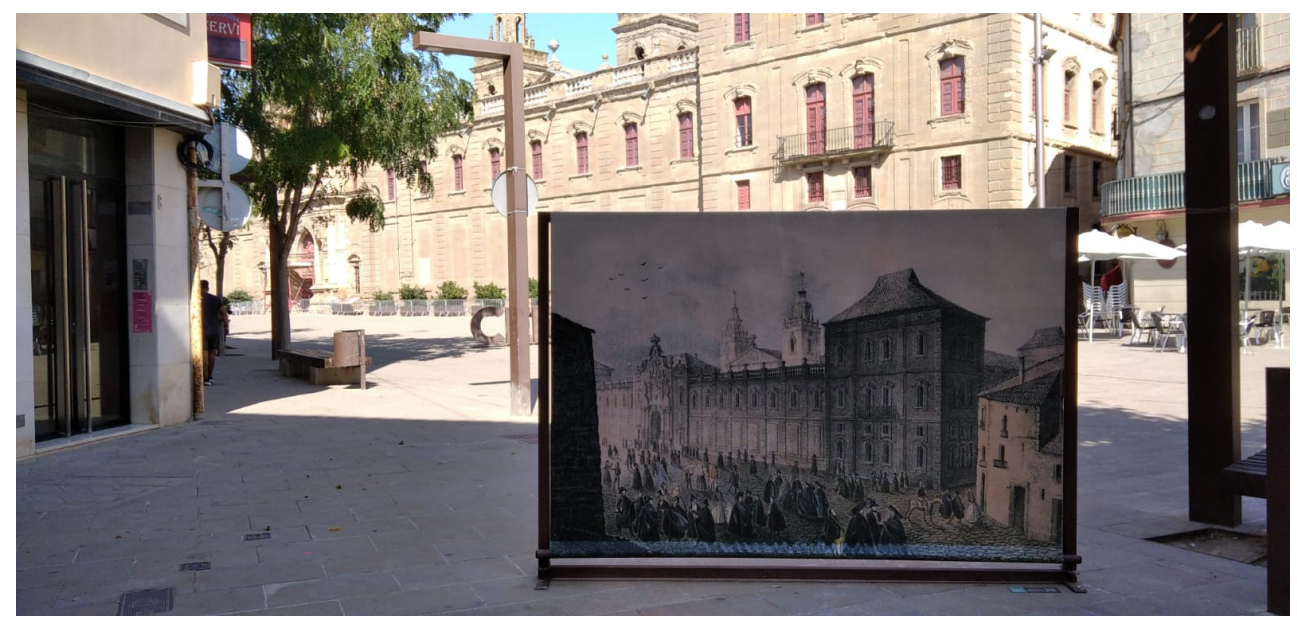

Fig. 7 Señalización interpretativa con la imagen histórica a gran formato de la antigua Universidad de Cervera

\subsection{De lo digital a lo presencial}

El confinamiento de la población durante la primera ola de la pandemia obligó a replantear la oferta cultural de los museos, que se volcaron en producir recursos en línea para mitigar el cierre de los edificios. Había que traspasar los muros e intentar llegar a los hogares.

Meses después, y ya a las puertas del verano, llegó la primera desescalada del confinamiento $\mathrm{y}$ todos los museos, abiertos por fin de nuevo, plantearon actividades para dar respuesta a las demandas de la comunidad local. El Museu de Cervera aprovechó la oportunidad para idear 
nuevas acciones y, curiosa o aparentemente en dirección inversa a lo habitual, reconvirtió un proyecto que había nacido en formato digital en acción presencial.

Las tres mujeres de las rutas virtuales cobraron vida y protagonizaron tres nuevas visitas teatralizadas. La historia de vida de cada una de ellas era tan potente, que no cabía duda de la pertinencia de esas rutas, que fueron, indiscutiblemente, un éxito de público a pesar de los aforos limitados y las medidas sanitarias aplicadas.

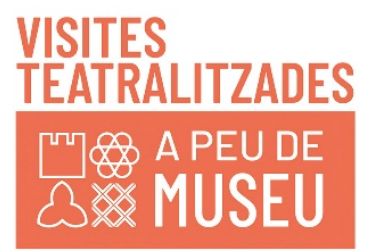

PREU 6€ /PREU REDUITT $4 €$ (de 8 al6 anys)

Gratuit per a menors de 8 anys

o museu@museudecervera.cat
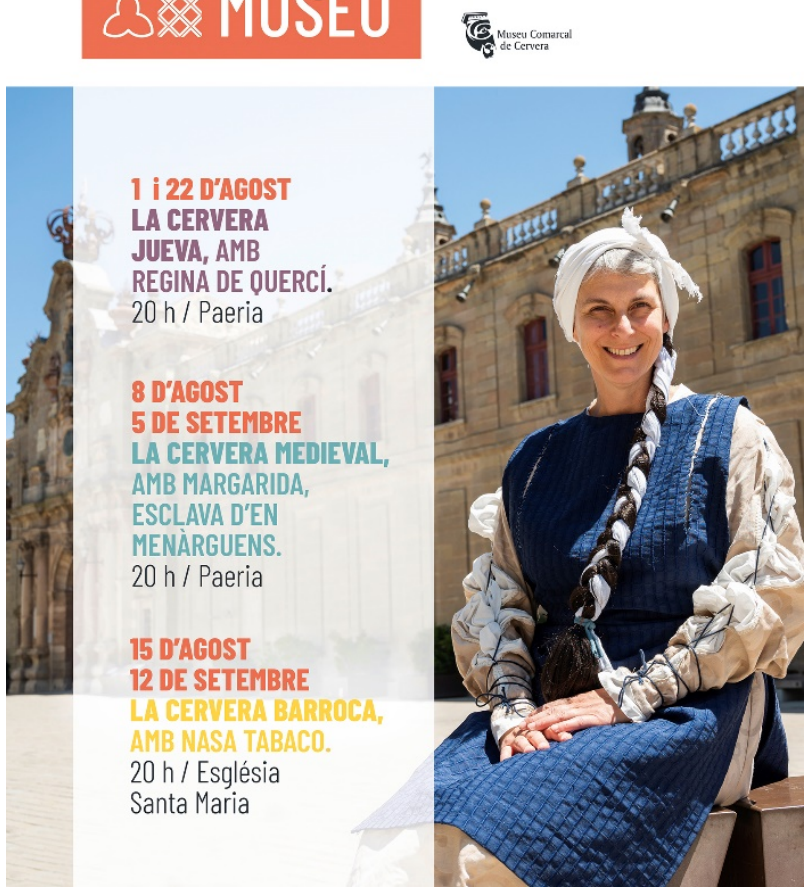

Fig. 8 Cartel editado para difundir las visitas teatralizadas 


\section{Resultados}

Parte indispensable del proceso de creación es la recogida y análisis de resultados, la evaluación, y el posible replanteamiento de aspectos susceptibles de mejora. Es decir, la evaluación entendida precisamente como proceso abierto y no como cierre del proyecto.

Nuestro aplicativo todavía tiene un recorrido corto y tan solo disponemos de las métricas relativas al periodo que va de septiembre de 2020 a febrero de 2021 — antes de esta fecha la útil herramienta de Google Analytic no funcionó por un error en la programación: siempre hay imponderables con los que hay que lidiar- Las cifras, más allá de valoraciones cuantitativas que, en este momento incipiente, aún no son relevantes, nos permiten plantear cuestiones importantes para mejorar el impacto del proyecto, conocer sus puntos débiles y proponer medidas correctoras que, en el momento del diseño inicial, sin aún usuarios reales, era imposible o muy difícil de predecir.

Podemos empezar, pues, a plantear preguntas y posibles respuestas. Algunas hacen referencia al propio sistema de recuento de datos, mientras que otras plantean conflictos en relación con los contenidos y la idoneidad de la señalización de los QR.

A modo de ejemplo, y a partir de una primera y rápida evaluación de las métricas, podemos empezar a hacernos algunas preguntas interesantes.

- En relación con el sistema de recuento:

¿Se contabilizan usuarios o visionados de contenidos? La verdad es que la información obtenida no permite distinguir cuántos usuarios visitan y finalizan la ruta. La información obtenida nos habla de los visionados de cada punto de interés, pero no de la fidelidad ni tampoco del acceso a los distintos apartados o menú de contenidos que aporta cada punto.

Por lo tanto, las cifras nos proporcionan, en este caso, una información fragmentaria, lo que nos plantea la necesidad de mejorar el sistema $\mathrm{y}$, al mismo tiempo, la posibilidad de implementar acciones complementarias que incentiven (y permitan conocer) la realización de la ruta completa.

- En relación con los contenidos y la señalización:

Las cifras nos muestran que hay una desigualdad muy evidente entre el visionado de un punto de interés de la ruta u otro. ¿Qué problemas pueden plantear los puntos menos visitados, que expliquen este bajo rendimiento? La respuesta puede ser doble: la señalización de los QR es deficiente, no tiene fácil acceso (ubicación equivocada) o se ve afectada por actos vandálicos (placas arrancadas); o el contenido del QR no resulta atractivo. Ambas respuestas precisarán de un análisis detallado para afrontar posibles soluciones. 
Tabla 2. Estadística extraída de Google Analytics

Perlode: Setembre 2020 - Febrer 2021

A PEU DE MUSEU:

\begin{tabular}{|c|c|}
\hline SECCIONS VISTIADES & PÀGIIES VISTES \\
\hline 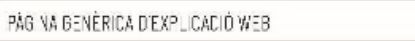 & 494 \\
\hline \multicolumn{2}{|l|}{ RUTA MEDIEVAL } \\
\hline Punt 1 - Castell de Cervera & 37 \\
\hline Punt 2 - Plaça Yella i Convent de St. Damènec & 17 \\
\hline Punt J-Església ce Santa Maria & 17 \\
\hline Punt 4 - Plaça cel Blat o Mercadal & 37 \\
\hline Punt 5 - Carreró de les Bruixes & 14 \\
\hline Punt 6 - Església de St. Bernat i casa de Santes Creus & 14 \\
\hline Punt 7 - El Call Jussà & 10 \\
\hline Punt 8 - Les Cases Medievals & 12 \\
\hline Punt 9 - Plaça de Santa Ánna o de les Bésties & 18 \\
\hline Punt 10 - Església de St Antoni & 25 \\
\hline TOTAL VISITES RUTA MEDIEVAL & 201 \\
\hline \multicolumn{2}{|l|}{ RUTA JUEVh } \\
\hline Punt1 - Carrers de conversos & 30 \\
\hline Punt 2 - Carrer dels Especiers & 11 \\
\hline Punt 3 -El Call Jussa & 20 \\
\hline Punt 4 - Placeta del Call i Torre dels Jueus & 14 \\
\hline Punt 5 - Carrer del Call & 8 \\
\hline Punt 6 - Carrer de Sant Francesc & 12 \\
\hline TOTAL VISITES RUTA JUEVA & 95 \\
\hline \multicolumn{2}{|l|}{ RUTA BAFROCA } \\
\hline Punt1- Església de Santa Maria & 15 \\
\hline Punt 2-La Paeria & 24 \\
\hline Punt 3 - Colegio de Educandas & 9 \\
\hline Punt 4 - Casa i Carreró Sabater & 27 \\
\hline Punt 5 - Coll.legi de Sant Carles & 14 \\
\hline Punt $\hat{6}-$ Casa Dalmases & 7 \\
\hline Punt 7 - Plaça de Santa Anna & $1 \mathrm{~B}$ \\
\hline Punt 8 - Casa Parroquial & 27 \\
\hline Punt 9 - Universitat & 65 \\
\hline Punt 10 - Hospital de Castelltort & 11 \\
\hline TOTAL VISITES RUTA BARROCA & 217 \\
\hline TOTAL VISITES & $1.007^{*}$ \\
\hline
\end{tabular}




\section{Conclusiones}

El proyecto "A peu de Museu" ha representado para el Museu de Cervera una oportunidad para experimentar, para repensarse y para plantear nuevas formas de comunicación y difusión. Una oportunidad para aportar, desde geografías simbólicamente alejadas de los grandes centros culturales, y desde espacios museísticos modestos, contenidos y formatos potentes y atractivos. Una oportunidad para reivindicar la necesidad de llevar a cabo investigaciones rigurosas que aporten no solo conocimiento sino también intencionalidad, ya que buscan provocar reflexión y pensamiento crítico. No en vano, las protagonistas son mujeres anónimas, que representan aspectos subalternos e históricamente invisibilizados.

En este contexto de reivindicación del territorio (periférico) y de la microhistoria como herramienta fundamental para el conocimiento, la tecnología se ha puesto al servicio de estos propósitos superiores. Pero a esta tecnología hay que conocerla bien, hay que entrar en el ámbito digital con estrategia y planificación y hay que moverse con dominio entre los lenguajes, cambiantes y diversos, que el mundo digital nos proporciona. Ya desde el inicio del proyecto, la máxima dificultad no fue tanto la investigación — parte plenamente integrada y consolidada en las formas de trabajo del museo - sino, precisamente, el conocimiento limitado del medio digital - aún extraño en nuestro día a día-. Así pues, el desarrollo de este proyecto reabrió el debate en torno a los perfiles profesionales de nuestros museos y a la sobrecarga de unos equipos pequeños que asumen funciones varias.

En definitiva, seguimos hablando de la función social del museo y de conceptos como el acceso al conocimiento y la creación de nuevos relatos más inclusivos; la tecnología tan solo (y sobre todo) puede aportar soluciones y facilitar acciones alineadas con los nuevos tiempos y hábitos de vida.

\section{Referencias}

ILMS (2009). Museums, Libraries and 21st Century Skills.

$<$ https://www.imls.gov/sites/default/files/publications/documents/21stcenturyskills.pdf $>$ [consulta: 15 de junio de 2021]

Plan Estratégico del Museo de Cervera, 2021-2025 (documento interno en fase de redacción).

RODÀ, C. (2012). "La hora 2.0 de Museo Picasso de Barcelona" en Museos y Redes Sociales. ICOM España Digital, Revista del Comité Español de ICOM, núm. 5, p. 10-17.

SIERRA, A. (2012). "Sembrar el mundo de cultura" en Museos y Redes Sociales. ICOM España Digital, Revista del Comité Español de ICOM, núm. 5, p. 58-67.

STACK, J. (2013). Tate Digital Strategy 2013-15: Digital as a Dimension of Everything, Tate Papers, núm. 19. <https://www.tate.org.uk/research/publications/tate- 
papers/19/tate-digital-strategy-2013-15-digital-as-a-dimension-of-everything> [consulta: 15 de junio de 2021]

TASICH, T. (2014). "Estrategias de transformación de los museos en la era digital. Exploramos estrategias prácticas que las instituciones podrían utilizar para hacer sus iniciativas de cambio más exitosas" en Estrategias digitales en los museos, dosier CCCBLab <https://lab.cccb.org/es/estrategias-de-transformacion-de-los-museos-en-laera-digital/> [consulta: 15 de junio de 2021] 